(C) The Authors 2017. This is an Open Access article, distributed under the terms of the Creative

Commons Attribution licence (http://creativecommons.org/licenses/by/4.0/), which permits unrestricted re-use,

distribution, and reproduction in any medium, provided the original work is properly cited.

\title{
Strawberry and cranberry polyphenols improve insulin sensitivity in insulin-resistant, non-diabetic adults: a parallel, double-blind, controlled and randomised clinical trial
}

\author{
Martine Paquette $^{1,2}$, Ana S. Medina Larqué ${ }^{1,2}$, S. J. Weisnagel ${ }^{2,3}$, Yves Desjardins ${ }^{1}$, Julie Marois ${ }^{1,2}$, \\ Geneviève Pilon $^{1,4}$, Stéphanie Dudonné ${ }^{1}$, André Marette ${ }^{1,4}$ and Hélène Jacques ${ }^{1,2_{*}}$ \\ ${ }^{1}$ Institute of Nutrition and Functional Foods, Laval University, Quebec, Canada, G1V OA6 \\ ${ }^{2}$ School of Nutrition, Laval University, Quebec, Canada, G1V OA6 \\ ${ }^{3}$ Diabetes Research Unit, Endocrinology and Nephrology Axis, Research Centre, Laval University Health Center of Quebec, \\ Quebec, Canada, G1V $4 G 2$ \\ ${ }^{4}$ Quebec Heart and Lung Institute, Quebec, Canada, G1V $4 G 5$
}

(Submitted 3 June 2016 - Final revision received 13 January 2017 - Accepted 28 January 2017 - First published online 14 March 2017)

\section{Abstract}

Plant-derived foods rich in polyphenols are associated with several cardiometabolic health benefits, such as reduced postprandial hyperglycaemia. However, their impact on whole-body insulin sensitivity using the hyperinsulinaemic-euglycaemic clamp technique remains under-studied. We aimed to determine the effects of strawberry and cranberry polyphenols (SCP) on insulin sensitivity, glucose tolerance, insulin secretion, lipid profile, inflammation and oxidative stress markers in free-living insulin-resistant overweight or obese human subjects ( $n$ 41) in a parallel, double-blind, controlled and randomised clinical trial. The experimental group consumed an SCP beverage (333 mg SCP) daily for 6 weeks, whereas the Control group received a flavour-matched Control beverage that contained 0 mg SCP. At the beginning and at the end of the experimental period, insulin sensitivity was assessed by a hyperinsulinaemic-euglycaemic clamp, and glucose tolerance and insulin secretion by a 2 -h oral glucose tolerance test (OGTT). Insulin sensitivity increased in the SCP group as compared with the Control group $\left(+0.9(\right.$ SEM 0.5$) \times 10^{-3} v .-0.5($ SEM 0.5$) \times 10^{-3} \mathrm{mg} / \mathrm{kg}$ per min per pmol, respectively, $\left.P=0.03\right)$. Compared with the Control group, the SCP group had a lower first-phase insulin secretion response as measured by C-peptide levels during the first 30 min of the OGTT $(P=0 \cdot 002)$. No differences were detected between the two groups for lipids and markers of inflammation and oxidative stress. A 6-week dietary intervention with $333 \mathrm{mg}$ of polyphenols from strawberries and cranberries improved insulin sensitivity in overweight and obese non-diabetic, insulin-resistant human subjects but was not effective in improving other cardiometabolic risk factors.

Key words: Polyphenols: Strawberries: Cranberries: Insulin sensitivity: Glucose metabolism: Insulin secretion: Insulin-resistant subjects

According to the International Diabetes Federation ${ }^{(1)}$, up to 592 million people worldwide (one in ten adults) will suffer from type 2 diabetes by the year 2035. This alarming increase has been predicted based on several factors, such as the high prevalence of obesity and sedentary lifestyles ${ }^{(2,3)}$. Indeed in obese humans, elevated levels of NEFA, pro-inflammatory cytokines and other factors produced by adipose tissue are key determinants of insulin resistance ${ }^{(4)}$. To maintain plasma glucose at normal levels, pancreatic $\beta$-cells must adjust their function to compensate for insulin resistance. This leads to an exhaustion of $\beta$-cell insulin secretion and the development of impaired glucose tolerance (IGT) and subsequent type 2 diabetes.

In recent decades, scientific evidence has shown a link between increased consumption of fruits and vegetables, particularly berries, and reduced incidence of type 2 diabetes ${ }^{(5)}$. Recent reviews have indeed reported that berries, like strawberries and cranberries, can lower markers of cardiometabolic risk ${ }^{(6-11)}$ and improve markers of the metabolic syndrome in humans ${ }^{(12-14)}$. It is well-documented that strawberries and cranberries are rich in polyphenols and contain a wide variety of phenolic compounds, ranging from phenolic acids (hydroxybenzoic and hydroxycinnamic acids), flavonoids (anthocyanins, flavonols and flavan-3-ols) to polymerised molecules (proanthocyanidins and ellagitannins) ${ }^{(15)}$. According to several in vitro and animal studies, polyphenols may improve glucose metabolism ${ }^{(16)}$ and peripheral glucose uptake in insulin-sensitive tissues by increasing GLUT4 translocation and activity and reducing oxidative stress and inflammation ${ }^{(17,18)}$. It has recently been demonstrated that anthocyanin-rich bilberry

Abbreviations: IAUC, incremental AUC; IGT, impaired glucose tolerance; $M / I$, insulin sensitivity index; OGTT, oral glucose tolerance test; SCP, strawberry and cranberry polyphenols; UHPLC, ultra-high performance liquid chromatography (UHPLC)

* Corresponding author: H. Jacques, fax +1 418656 3353, email helene.jacques@fsaa.ulaval.ca 
extract reduces glycaemia and improves insulin sensitivity in diabetic mice ${ }^{(19)}$. Afrin et al. ${ }^{(12)}$ reviewed six human intervention studies on the impact of strawberries on the metabolic syndrome, and specifically on the prevention of type 2 diabetes, but none used the gold standard clamp technique to assess insulin sensitivity.

There are several indices calculated from the oral glucose tolerance test (OGTT), such as the Matsuda index and insulin sensitivity index (ISI), or from fasting glycaemia and/or insulinaemia, such as the homeostasis model assessment of insulin sensitivity (HOMA-IR) and the quantitative insulin sensitivity check index (QUICKI), that indirectly estimate insulin sensitivity in humans. However, these indices are not as accurate as a direct measurement of whole-body insulin sensitivity by the hyperinsulinaemic-euglycaemic clamp ${ }^{(20)}$. In this respect, the clamp technique is recognised as the reference method for measuring insulin sensitivity, and according to Antuna-Puente et $a l .{ }^{(21)}$, it should be promoted in clinical human studies. So far, only two studies have used a hyperinsulinaemic-euglycaemic clamp to assess the effect of berry polyphenols on insulin sensitivity ${ }^{(22,23)}$. According to one study in which obese non-diabetic insulinresistant participants received a blueberry or placebo smoothie twice a day, the mean percentage increase in insulin sensitivity was five times greater in the experimental group compared with that in the placebo group ${ }^{(22)}$. In the second study, a grape polyphenol supplement protected against a decrease in insulin sensitivity caused by a fructose-rich diet in overweight subjects ${ }^{(23)}$. To the best of our knowledge, there are no human studies evaluating the effects of strawberry and cranberry polyphenols (SCP) on insulin sensitivity assessed by the hyperinsulinaemic-euglycaemic clamp in non-diabetic insulin-resistant subjects.

The proposed study aims to test the effects of an SCP blend incorporated in a beverage on insulin sensitivity and related parameters in free-living overweight or obese men and women with insulin resistance. This blend has been comprehensively analysed and contains well-defined amounts of anthocyanins, proanthocyanidins, ellagitannins, phenolic acids and flavonols (quercetins) $^{(24)}$. The primary endpoint was the difference in the change in insulin sensitivity after 6 weeks of SCP consumption compared with a SCP-free Control beverage. Secondary endpoints aimed to assess changes in glucose tolerance, insulin secretion, plasma lipids, markers of oxidative stress and inflammation, as well as to characterise plasma phenolic components as bioavailability (efficacy) outcomes. We hypothesised that the consumption of SCP improves insulin sensitivity and lipid profile and reduces inflammatory and oxidative stress markers in overweight/obese subjects.

\section{Methods}

\section{Study design}

This 6-week clinical trial was a randomised, double-blinded, controlled, parallel study performed at the Institute of Nutrition and Functional Foods (INAF) in Quebec City, Canada. The study was conducted in accordance with the Declaration of Helsinki and all procedures involving human subjects were approved by the Research Ethical Committee of the Quebec University Health Center. Written informed consent was obtained from all participants after reading a detailed consent form prior to their participation in the study. This trial was registered at clinicaltrials.gov as NCT01766570. The study was conducted, including analyses, between spring 2012 and fall 2015

\section{Subjects}

All subjects were overweight or obese $\left(\mathrm{BMI} \geq 25 \mathrm{~kg} / \mathrm{m}^{2}\right)$ and insulin resistant based on fasting plasma insulin level $>60 \mathrm{pmol} / \mathrm{l}$ (J.-P. Després and J. Bergeron, unpublished results). Subjects may have also displayed impaired fasting plasma glucose (IFG) (5.6-6.9 mmol/l) with or without IGT $(7.8-11.0 \mathrm{mmol} / \mathrm{l})$ following a $2-\mathrm{h} 75 \mathrm{~g}$ OGTT $^{(25)}$. Exclusion criteria included smoking, chronic disease (diabetes, respiratory, renal, gastrointestinal or hepatic disease, CVD, hypertension, cancer), metabolic or acute disease, use of medication or supplement known to affect lipid or glucose metabolism, use of antioxidant supplements, major surgery in the 3 months preceding the study and significant weight change (sEm 10\%) within 6 months prior to beginning the study.

A total of 116 subjects, recruited in the Quebec City metropolitan area by media advertising, were first screened to examine their eligibility to participate in this study. Of the fifty eligible subjects who began the experimental period, forty-six participants (twenty men and twenty-six women) aged between 40 and 70 years completed the study. However, five subjects (three in the SCP group and two in the Control group) were excluded from analysis because they no longer met some inclusion criteria, leading to a total of eighteen men (nine in each of the two groups) and twenty-three postmenopausal women (eleven in the SCP group and twelve in the Control group) who were included in the statistical analysis (online Supplementary Fig. S1).

\section{Experimental groups}

The polyphenol blend and Control were provided as energy-free beverages formulated with purified water and small amounts of sucralose. Red food colour was added in the Control beverage. Both beverages thus had the same taste and visual aspect. Both were formulated in a single batch by Atrium Innovations Inc. and were provided in dark bottles at $120 \mathrm{ml} / \mathrm{d}$. The bottles were sealed and kept at room temperature with limited light exposure. The polyphenol blend contained $1.84 \mathrm{~g}$ of a mixture of dry strawberry (Fragaria $\times$ ananassa Duch) and cranberry (Vaccinium macrocarpon L.) polyphenol extracts (GlucoPhenol ${ }^{\mathrm{TM}}$; NutraCanada) providing an average daily dose of 333 (SD 12) $\mathrm{mg}$ of polyphenols (18\% total polyphenols in GlucoPhenol ${ }^{\mathrm{TM}}$, as determined by Folin-Ciocalteu assay). SCP (GlucoPhenol ${ }^{\mathrm{TM}}$ ) complies with good manufacturing practices according to Health Canada regulations. To determine the amount of polyphenols to be used for the present study, we first conducted a bioavailability study in rats with different doses of $\mathrm{SCP}^{(24)}$. The dose of $333 \mathrm{mg}$ polyphenols was chosen as corresponding, for a $60 \mathrm{~kg}$ human, to the dose $(36 \mathrm{mg} / \mathrm{kg})$ that induced the highest concentration of plasma total phenolic metabolites in the rat ${ }^{(24)}$. The total phenolic content was monitored in the SCP beverage throughout the study, ensuring a minimum of $300 \mathrm{mg} / \mathrm{d}$. In order to simulate the taste 
Table 1. Phenolic composition of experimental beverages (Mean values and standard deviations)

\begin{tabular}{|c|c|c|c|c|}
\hline \multirow[b]{2}{*}{ Phenolic composition $(\mu \mathrm{g} / 120 \mathrm{ml})$} & \multicolumn{2}{|c|}{ SCP } & \multicolumn{2}{|c|}{ Control } \\
\hline & Mean & SD & Mean & SD \\
\hline Proanthocyanidins & $20040^{\star}$ & 522 & 5070 & 169 \\
\hline Monomers & $4156^{*}$ & 9 & 1276 & 36 \\
\hline Dimers & 237 & 5 & ND & ND \\
\hline Polymers & $15647^{\star}$ & 528 & 3794 & 133 \\
\hline Phenolic acids & $28206^{*}$ & 256 & 5013 & 138 \\
\hline Gallic acid & $728^{*}$ & 5 & 11 & 2 \\
\hline Hydroxybenzoic acid & $3334^{*}$ & 94 & 103 & 12 \\
\hline$p$-Coumaric acid & $9095^{\star}$ & 171 & 47 & 9 \\
\hline$m$-Coumaric acid & $2051^{*}$ & 80 & 6 & 1 \\
\hline Caffeic acid & 372 & 22 & ND & ND \\
\hline Ferulic acid & $65^{\star}$ & 7 & 33 & 4 \\
\hline Vanillic acid & $107^{*}$ & 3 & 3 & 0.3 \\
\hline Caffeoyl glucoside & $1282^{*}$ & 54 & 8 & 2 \\
\hline Coumaroyl glucoside & $5508^{*}$ & 178 & 9 & 4 \\
\hline Chlorogenic acid & $927^{*}$ & 14 & 240 & 28 \\
\hline
\end{tabular}

SCP, strawberry and cranberry polyphenols; ND, not detectable/below limit of detection.

The SCP beverage provided an average daily dose of 333 (SD 12) $\mathrm{mg}$ of polyphenols, as determined by Folin-Ciocalteu assay.

* Welch's $t$ test and the Mann-Whitney test showed significant differences $(P<0.05)$ in concentrations of each measured phenolic compound in SCP beverage compared with Control.

and colour of the SCP-containing beverage, a pomegranatederived red food colour was used in the SCP-free Control beverage providing a small quantity of polyphenols. The two beverages were characterised for their phenolic composition as previously described $^{(24)}$ (Table 1). In brief, proanthocyanidins were analysed by normal-phase HPLC with fluorescence detection, and quantified using an epicatechin standard. Phenolic acids were analysed using reverse-phase ultra-high performance liquid chromatography (UHPLC) coupled with tandem MS and their quantification was achieved using their corresponding standard when available, or their aglycone or most similar phenolic structure otherwise. The daily phenolic dose provided by SCP corresponds approximately to the intake of $112 \mathrm{~g}$ of fresh fruit (equivalent to two servings).

\section{Study timeline}

Participants were equally divided into two groups by sex after a 2-week run-in period. During this period, subjects were asked to maintain their usual food habits and physical activity level, and were limited to one unit drink or less of beer or spirits per day. The consumption of berries, wine, polyphenol supplements and all products containing berries or wine was also forbidden. Assignment of treatment was conducted through the use of a random sequence of numbers. Allocation to treatment was concealed by a secure computer-assisted method enabling preservation of assignments until enrolment was assured and confirmed. Men and women were equally distributed among the two groups using the same computer-assisted method. The study sponsor held the trial codes which were disclosed after completion of the statistical analyses. Participants in the treatment group consumed an SCP-containing beverage, whereas the Control group received a flavour-matched SCP-free Control beverage, daily for a 6-week period. During the experimental period, subjects were asked to follow the same aforementioned recommendations as during the run-in period. Approximately
2-3 weeks after the beginning of the experimental period, a registered dietitian called all participants once to ensure that they consumed the beverages daily and followed the prescribed instructions. Subjects were instructed to shake the bottle before consumption and drink the beverage with or without food regardless of the time of day. To document compliance, subjects were requested to bring back the unused bottles at the end of the study. Bottle counts indicated a $99 \%$ compliance in both groups. Also, a 6-week checklist was provided to all participants to identify study materials that had not been ingested, thus providing us with a tool to confirm the compliance rate of the participants.

\section{Anthropometric and blood pressure measurements}

Fasted body weight, height, waist and hip circumferences were measured at the beginning and at the end of the study using standardised methods. BMI and waist:hip ratio were then calculated. Blood pressure was measured three times on the right arm with an automatic tensiometer (Digital Blood Pressure Monitor, model HEM-907XL; OMRON ${ }^{\circledR}$ ) following a 10-min rest at the beginning and at the end of the experimental period.

\section{Food records and questionnaires}

During the screening visit, two online self-administered questionnaires were completed by all subjects to collect information on medical history, lifestyle, economic and socio-demographic characteristics. Participants were also asked to complete two online self-administered questionnaires at the beginning and at the end of the experimental period, including a validated FFQ to record energy and macronutrient intake for 28 consecutive days $^{(26)}$ and a short physical activity questionnaire. There was also an additional questionnaire on the subjects' liking of the experimental beverages (taste, texture, etc.) and on side effects, administered at the end of the study. Changes in medication, 
temporary medication, natural health products intake or consumption of any other food supplements were monitored according to the exclusion criteria during the entire study period.

\section{Hyperinsulinaemic-euglycaemic clamp}

A 120-min hyperinsulinaemic-euglycaemic clamp was performed at the beginning and at the end of the experimental period at the Diabetes Research Unit of the Laval University Health Center of Quebec after a 12-h overnight fast, according to the method described by Piche et $a l .{ }^{(27)}$. Alcohol intake was forbidden $48 \mathrm{~h}$ before the clamp. Insulin sensitivity $(M / I)$ was calculated from glucose infusion rate $(\mathrm{mg} / \mathrm{min})$ during the final 30 min of the clamp divided by body weight $(\mathrm{kg})$ and then divided by the mean insulin concentration during the final 30 min of the clamp $(\mathrm{mg} / \mathrm{kg} \text { per min per } \mathrm{pmol})^{(27)}$. For NEFA analysis, additional blood samples were collected during the clamp at 0, 30, 60, 90 and $120 \mathrm{~min}$, centrifuged after $30 \mathrm{~min}$ at room temperature and then stored at $-80^{\circ} \mathrm{C}$ until analysis.

\section{Oral glucose tolerance test}

A 75-g OGTT was performed 2-3d before each clamp at the beginning and at the end of the experimental period at INAF to assess glucose tolerance after a 12-h overnight fast. Blood samples were collected at time points $-15,0,15,30,60$ and $120 \mathrm{~min}$, immediately centrifuged and kept at $-20^{\circ} \mathrm{C}$ for further measurements of glucose, insulin and C-peptide. Alcohol intake was forbidden $48 \mathrm{~h}$ before the test. For the second clamp and OGTT, participants were asked to consume the beverage $12 \mathrm{~h}$ before their appointment.

\section{Blood collection and storage}

Plasma and serum samples were collected in the fasting state, before each OGTT and clamp, and stored at $-80^{\circ} \mathrm{C}$ for further analysis of lipids, inflammatory markers (high-sensitivity C-reactive protein (hsCRP), IL-6, TNF- $\alpha$, high molecular weight (HMW) adiponectin and regulated on activation normal $\mathrm{T}$ cell expressed and secreted (RANTES)/chemokine ligand 5 (CCL5)), plasminogen activator inhibitor-1 (PAI-1), a marker of cardiovascular risk, and ferric reducing antioxidant power (FRAP) and oxidised LDL, markers of oxidative stress.

\section{Glucose, insulin and C-peptide}

Plasma glucose was determined using an enzymatic method ${ }^{(28)}$ and plasma insulin was measured by RIA with polyethylene glycol separation ${ }^{(29)}$. Plasma C-peptide level, an indicator of insulin secretion used to estimate pancreatic $\beta$-cell function, was determined using a modified version of the method of Hedging with polyclonal antibody A-4741 from Ventrex and polyethylene glycol precipitation ${ }^{(29)}$.

\section{Lipids}

LDL and HDL were isolated from fresh blood by ultracentrifugation combined with a heparin-manganese chloride precipitation $^{(30)}$. Then, cholesterol and TAG concentrations in total serum and lipoproteins were determined enzymatically by using a Technicon RA-500 analyzer (Bayer). NEFA were determined in serum via an enzymatic colorimetric assay (Wako Diagnostics) using a Beckman Olympus AU400 (Beckman Coulter Canada LP).

\section{Inflammatory, thrombogenic and oxidative markers}

Serum level of hsCRP was measured using nephelometry as described previously ${ }^{(31)}$. PAI-1, IL-6 and TNF- $\alpha$ were measured in plasma at the Quebec Heart and Lung Institute, Quebec, using commercially available Multiplex kits (EMD Millipore). Plates were read and analysed using the Bio-Plex 200 system (Bio-Rad). Oxidised-LDL, HMW adiponectin and RANTES were determined using a commercially available ELISA (Mercodia; R\&D Systems) according to manufacturer's instructions. Total antioxidant capacity of plasma, assessed by FRAP assay, was determined as described previously ${ }^{(32)}$.

\section{Bioavailability study}

A subgroup of seventeen subjects performed an additional bioavailability study, to identify circulating phenolic metabolites following the administration of experimental beverages. Halfway through the supplementation period (30 (SEM 3) d), fasted subjects were administered their respective treatment at INAF (SCP, $n$ 8; Control, $n$ 9). Blood samples were collected using EDTAcontaining syringes before and 30, 60, 120, 240 and 360 min after the ingestion. During the experiment, all subjects were kept fasted. Plasma samples were obtained by centrifugation $(3500 \mathrm{rpm}$, $10 \mathrm{~min}$ at $4^{\circ} \mathrm{C}$ ). Plasma phenolic compounds were characterised by UHPLC-MS/MS as previously described ${ }^{(24)}$, with slight modifications. Acidified plasma samples $(300 \mu \mathrm{l})$ were loaded into preconditioned Waters OASIS HLB (Waters Ltd) $\mu$ Elution plates $2 \mathrm{mg}-30 \mu \mathrm{m}$. The retained phenolic compounds were eluted with $75 \mu$ l of acetone-ultrapure water-acetic acid solution (70:29.5:0.5, $\mathrm{v} / \mathrm{v} / \mathrm{v})$ in presence of rosmarinic acid as internal standard $(1 \mu \mathrm{g} / \mathrm{ml}$ final concentration). The eluted solutions were directly analysed by UHPLC-MS/MS, using a Waters Xevo TQD MS (Waters Ltd) coupled to a Waters Acquity UHPLC (Waters Ltd). Phenolic metabolites were separated and identified as previously reported $^{(24)}$.

\section{Statistical analyses}

We estimated sample size based on the primary endpoint of insulin sensitivity from data published by Stull et al. ${ }^{(22)}$ and Ouellet et $a l .{ }^{(33)}$. For this purpose, we used the following values: an average difference in changes from baseline (Post $v$. Pre) of $17 \times 10^{-3} \mathrm{mg} / \mathrm{kg}$ per min per pmol for insulin sensitivity between the SCP and Control groups after 6 weeks and an estimated SD of 18 . Power calculation at $80 \%$ with a two-sided significance level set at 0.05 showed that a minimum of forty subjects, twenty in each group, was required to observe significant changes from baseline in insulin sensitivity between the SCP and Control groups over a 6-week dietary intervention, taking into account $25 \%$ expected dropouts. Statistical analyses were performed using SAS 9.3 (SAS Institute).

Paired $t$ tests were performed to compare changes from baseline (Post $v$. Pre) within the same group. Because baseline 
insulin sensitivity was correlated with $M / I\left(r_{\mathrm{s}}=0.50 ; n 39\right.$; $P<0.001)$ only, PROC MIXED for ANCOVA with baseline insulin sensitivity as covariate was used to compare the changes in $M / I$ between the two treatments. A two-way repeatedmeasures ANOVA was applied for variables with repeated measures over time (glucose, insulin, C-peptide and NEFA concentrations during the OGTT or clamp). In this model, no significant time-by-treatment interaction was observed. Furthermore, positive incremental AUC (IAUC) for glucose (mmol/l per $\mathrm{min}$ ), insulin ( $\mathrm{pmol} / \mathrm{l}$ per $\mathrm{min}$ ) and C-peptide (pmol/l per min) up to 30 and 120 min were calculated using the trapezoid method with baseline value corresponding to the fasting level (time point $-15 \mathrm{~min}$ of the OGTT). The percentage change in IAUC ((IAUC post value - IAUC pre value $) \times 100$ / IAUC pre value) was also calculated. PROC MIXED for a two-way ANOVA was used to compare changes from baseline for anthropometric and blood pressure measurements, IAUC glucose, insulin and C-peptide during OGTT, lipid and cardiovascular parameters and markers of inflammation and oxidative stress. Data from men and women were pooled together because there was no significant sex-by-treatment interaction. As several significant correlations were observed within and between OGTT and lipid variables, a Bonferroni correction was applied, setting the two-sided significance level at $P<0.004$ for those variables. No Bonferroni correction was applied for $M / I$, inflammatory and oxidative stress because no correlation was observed between those and the other variables, and a two-sided significance level was set at 0.05. PROC GLM ANOVA was used to compare the FFQ variables.

Plasma concentrations of phenolic metabolites were measured post-ingestion in a subgroup ( $n$ 17) and were compared using the Welch's $t$ test (correcting for unequal variance) when data were assumed to be normally distributed (or met the criteria for normality after log transformation), or using the non-parametric Mann-Whitney test otherwise, using GraphPad Prism 6.05 software. Correlations were assessed between circulating concentrations of phenolic metabolites (expressed as area under the plasma concentration (nM) time (min) curve between 0 and $30 \mathrm{~min}$ after the ingestion of the beverages) and changes in $M / I$ and in outcomes of OGTT (expressed as the percentage change in the IAUC for glucose, insulin and C-peptide during the first $30 \mathrm{~min}$ of the OGTT). A robust regression with $M$-estimations was adjusted using the procedure ROBUSTREG of SAS 9.4. To perform an optimal selection of the model, the smallest Akaike criterion was deemed the best model. Considering the small size of the subgroup and the multiplicity of analysis, a Bonferroni correction $(P<0 \cdot 0025)$ was performed for post-ingestion plasma concentration of phenolic metabolites. Bonferroni correction was also applied for the correlational analysis with the outcomes of the first $30 \mathrm{~min}$ OGTT parameters $(P<0 \cdot 017)$. Since $M / I$ assesses insulin sensitivity by hyperinsulinaemic-euglycaemic clamp, correlations with $M / I$ changes were analysed separately from the OGTT rate changes and remained at a statistically significant level of $P \leq 0.05$. The results are presented as means with their standard errors.

\section{Results}

\section{Subject baseline characteristics}

Baseline clinical and laboratory characteristics of participants are shown in Table 2. All subjects were insulin resistant, overweight or obese (BMI $\geq 25 \mathrm{~kg} / \mathrm{m}^{2}$ ) with increased abdominal adiposity (waist circumference $>94 \mathrm{~cm}$ for men and $>80 \mathrm{~cm}$ for women). There were no differences between the two groups

Table 2. Baseline characteristics of the study participants* (Mean values with their standard errors; numbers and percentages)

\begin{tabular}{|c|c|c|c|c|c|}
\hline \multirow[b]{2}{*}{ Variables } & \multicolumn{2}{|c|}{$\operatorname{SCP}(n 20)$} & \multicolumn{2}{|c|}{ Control $(n 21)$} & \multirow[b]{2}{*}{$P$} \\
\hline & Mean & SEM & Mean & SEM & \\
\hline Age (years) & 57 & 1 & 60 & 1 & 0.18 \\
\hline Body weight $(\mathrm{kg})$ & 85 & 3 & 85 & 3 & 0.97 \\
\hline BMI $\left(\mathrm{kg} / \mathrm{m}^{2}\right)$ & 31 & 1 & 31 & 1 & 0.91 \\
\hline Waist circumference $(\mathrm{cm})$ & 104 & 3 & 104 & 2 & 0.95 \\
\hline Hip circumference $(\mathrm{cm})$ & 111 & 2 & 111 & 2 & 0.93 \\
\hline \multicolumn{6}{|l|}{ Cholesterol (mmol/l) } \\
\hline Total & $5 \cdot 70$ & 0.17 & $5 \cdot 37$ & 0.22 & 0.07 \\
\hline HDL & 1.25 & 0.05 & 1.33 & 0.05 & 0.24 \\
\hline LDL & 3.52 & 0.17 & $3 \cdot 20$ & 0.15 & 0.18 \\
\hline Total TAG (mmol/l) & 2.03 & 0.24 & 1.73 & 0.26 & 0.39 \\
\hline Total cholesterol:HDL-cholesterol ratio & $4 \cdot 8$ & 0.3 & $4 \cdot 1$ & 0.2 & 0.07 \\
\hline Fasting plasma glucose $(\mathrm{mmol} / \mathrm{l})$ & $6 \cdot 0$ & 0.1 & 5.8 & $0 \cdot 1$ & 0.16 \\
\hline 2-h plasma glucose (mmol/l) & $7 \cdot 7$ & 0.4 & 7.4 & 0.4 & 0.71 \\
\hline \multirow[t]{2}{*}{ Fasting plasma insulin (pmol/l) } & 118 & 11 & 130 & 11 & 0.45 \\
\hline & $n$ & $\%$ & $n$ & $\%$ & \\
\hline \multicolumn{6}{|l|}{ Sex } \\
\hline Women & 11 & 55 & 12 & 57 & - \\
\hline Men & 9 & 45 & 9 & 43 & - \\
\hline
\end{tabular}

SCP, strawberry and cranberry polyphenols.

* PROC MIXED ANOVA test showed no significant differences in baseline characteristics between the two groups. 
regarding age, body weight, BMI, waist and hip circumferences, lipid profile, fasting plasma glucose, 2-h plasma glucose or fasting plasma insulin.

At baseline, all subjects had a high fasting plasma insulin level ( $>60 \mathrm{pmol} / \mathrm{l}$ ), of whom thirty-one had fasting plasma insulin levels $>90 \mathrm{pmol} / \mathrm{l}$. From data collected during the preintervention OGTT and according to the Expert Committee on the Diagnosis and Classification of Diabetes Mellitus ${ }^{(25)}$, twelve subjects had both IFG $(5 \cdot 6-6.9 \mathrm{mmol} / \mathrm{l})$ and IGT $\left(7 \cdot 8_{-}\right.$ $11.0 \mathrm{mmol} / \mathrm{l})$, seventeen subjects had IFG only, three subjects had IGT only and nine among them had normal glucose tolerance (fasting plasma glucose $<5.6 \mathrm{mmol} / 1$ and plasma glucose $<7.8 \mathrm{mmol} / 1$ after $120 \mathrm{~min}$ ).

\section{Food intake and physical activity}

According to FFQ data (online Supplementary Table S1), there were no differences in baseline dietary intake or in changes from baseline (Post $v$. Pre) in energy and macronutrient intake between the two groups. In addition, no change from the level of physical activity was perceived during the study (data not shown). As for side effects, no major harmful or unexpected effects were reported in either group.

\section{Anthropometric measurements and blood pressure}

Body weight, anthropometric, systolic and diastolic blood pressure measurements were performed at the beginning and at the end of the experimental period. No differences in changes from baseline (Post $v$. Pre) were observed for these parameters between the two groups (online Supplementary Table S2).

\section{Insulin sensitivity and other parameters of glucose homoeostasis}

Insulin sensitivity $(M / D)$ increased by $14 \%\left(+0.9(\right.$ SEM 0.5$) \times 10^{-3} \mathrm{mg} /$ $\mathrm{kg}$ per min per pmol) (Fig. 1) in the SCP group $(P=0.05)$, whereas it decreased by $7 \%(-0.5$ (SEM 0.5$) \times 10^{-3} \mathrm{mg} / \mathrm{kg}$ per min per pmol) in the Control group without achieving a level of significance $(P=0 \cdot 28)$. When we compared the changes from baseline (Post $v$. Pre) between the two treatments, the SCP group showed significant improvement in insulin sensitivity $(P=0.03)$ compared with Control.

We also performed repeated measurements ANOVA for glucose (Table 3), insulin (Table 3) and C-peptide (Fig. 2(a) and (b)) up to $120 \mathrm{~min}$ during OGTT and for NEFA (online Supplementary Fig. S2) over time during the clamp. There were no differences between baseline values (Pre) for all glucose metabolism parameters. Whereas glucose, insulin and NEFA responses were not different between treatments, there was an overall increase in C-peptide with Control compared with SCP $(P=0.002)$ (Fig. 2(c)).

The mean IAUC up to $30 \mathrm{~min}$ corresponding to the early phase of insulin response during the OGTT and the mean IAUC up to $120 \mathrm{~min}$ after the OGTT are shown in Table 3 for plasma glucose and insulin and in Fig. 3 and 4 for C-peptide. No differences in changes from baseline (Post $v$. Pre) for plasma IAUC glucose (Table 3), IAUC insulin (Table 3) and IAUC C-peptide (Fig. 3) were observed up to 120 min within each group or

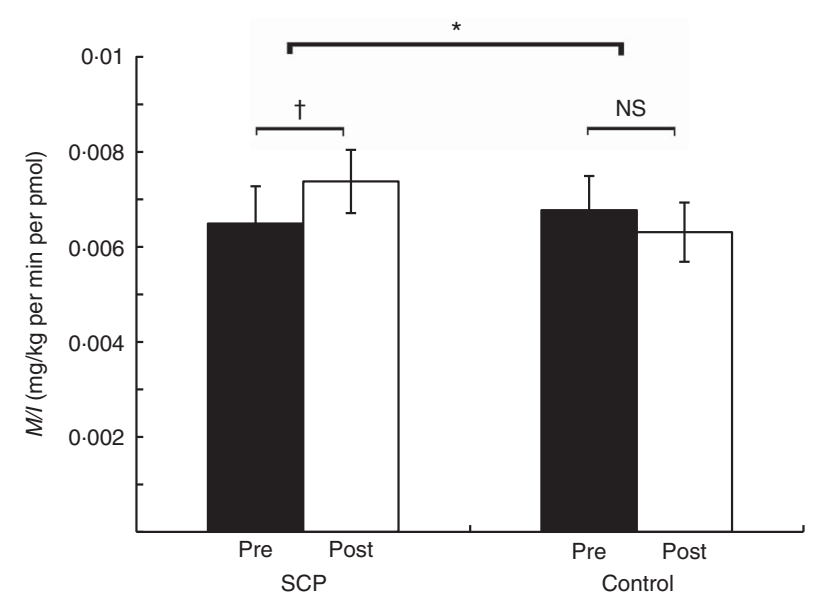

Fig. 1. Insulin sensitivity $(M / I)$ before (Pre) and after (Post) 6-week consumption of strawberry and cranberry polyphenols (SCP) or Control in insulin-resistant human subjects. Values are means ( $n$ 39), with their standard errors represented by vertical bars. NS, no significant difference. Paired $t$ test for comparisons from baseline (Post $v$. Pre) within each group showed a significant increase with SCP, $† P=0.05$. PROC MIXED for ANCOVA with baseline insulin sensitivity as covariate indicated significant difference in changes from baseline (Post $v$. Pre) between the two groups, ${ }^{*} P=0.03$.

between the two groups. However, compared with the baseline (Pre) values, plasma IAUC C-peptide up to $30 \mathrm{~min}$ was increased by $26 \%$ in the Control group $(P=0.003)$ and non-significantly reduced by $8 \%$ in the SCP group $(P=0 \cdot 21)$. These changes were different between the two groups ( $P=0.002)$ (Fig. 4).

\section{Lipid profile}

No differences in changes from baseline (Post $v$. Pre) for total, LDL- and HDL-cholesterol or TAG were observed within each group or between the two groups (Table 4).

\section{Inflammatory, thrombogenic and oxidative markers}

The effects of SCP on inflammatory and oxidative stress markers are shown in Table 4. No differences in changes from baseline (Post $v$. Pre) for pro-inflammatory cytokines, hsCRP, HMW adiponectin, PAI-1, oxidised-LDL, RANTES or total antioxidant capacity of plasma (FRAP) were observed within each group or between the two groups.

\section{Phenolic composition and bioavailability of experimental beverages}

As shown in Table 1, SCP-containing and SCP-free Control beverages differed significantly in terms of phenolic content. SCP contained four times more proanthocyanidins and six times more phenolic acids. In particular, SCP were characterised by a very high content of coumaric acids: $p$-coumaric acid, $m$-coumaric acid and coumaroyl glucoside. At the 30-d midpoint of the daily consumption of the beverages, about twenty phenolic metabolites were identified in the plasma of volunteers. They are present in circulation as conjugate metabolites and microbial degradation products, because the native phenolic compounds are normally extensively metabolised. 
Table 3. Incremental AUC (IAUC) and time point values over time during oral glucose tolerance test (OGTT) for glucose and insulin before and after 6-week consumption of strawberry and cranberry polyphenols (SCP) or Control in insulin-resistant human subjects (Mean values with their standard errors)

\begin{tabular}{|c|c|c|c|c|c|c|c|c|c|c|c|}
\hline \multirow[b]{3}{*}{ Variables } & \multicolumn{4}{|c|}{$\operatorname{SCP}(n 20)$} & \multirow[b]{3}{*}{$P_{1}^{*}$} & \multicolumn{5}{|c|}{ Control (n 21) } & \multirow[b]{3}{*}{$P \dagger$} \\
\hline & \multicolumn{2}{|c|}{ Pre } & \multicolumn{2}{|c|}{ Post } & & \multicolumn{2}{|c|}{ Pre } & \multicolumn{2}{|c|}{ Post } & \multirow[b]{2}{*}{$P_{2}^{*}$} & \\
\hline & Mean & SEM & Mean & SEM & & Mean & SEM & Mean & SEM & & \\
\hline IAUC glucose up to $30 \mathrm{~min}(\mathrm{mmol} / / \mathrm{per} \mathrm{min})$ & 56 & 4 & 58 & 6 & 0.77 & 55 & 5 & 53 & 4 & 0.48 & 0.77 \\
\hline Plasma glucose $(\mathrm{mmol} / \mathrm{l})$ & & & & & 0.42 & & & & & 0.53 & $0.31 \ddagger$ \\
\hline-15 & $6 \cdot 1$ & 0.1 & $6 \cdot 1$ & 0.1 & & 5.9 & 0.1 & $6 \cdot 0$ & 0.1 & & \\
\hline 0 & 6.0 & 0.1 & $6 \cdot 1$ & 0.1 & & 5.8 & 0.1 & 5.9 & 0.1 & & \\
\hline 15 & 8.1 & 0.2 & $8 \cdot 1$ & 0.3 & & 7.7 & 0.2 & 8.0 & 0.2 & & \\
\hline 30 & 9.7 & 0.3 & 9.8 & 0.3 & & $9 \cdot 3$ & 0.3 & 9.4 & 0.3 & & \\
\hline 60 & $10 \cdot 3$ & 0.5 & 10.6 & 0.4 & & 9.9 & 0.5 & 9.5 & 0.4 & & \\
\hline 120 & $7 \cdot 7$ & 0.4 & 7.5 & 0.4 & & 7.4 & 0.4 & 6.9 & 0.3 & & \\
\hline IAUC glucose up to $120 \mathrm{~min}(\mathrm{mmol} / / \mathrm{per} \mathrm{min})$ & 348 & 31 & 357 & 32 & 0.71 & 329 & 34 & 291 & 27 & 0.11 & $0 \cdot 16$ \\
\hline IAUC insulin up to $30 \mathrm{~min}(\mathrm{pmol} / \mathrm{l}$ per $\mathrm{min})$ & 9 & 1 & 8 & 1 & 0.56 & 10 & 1 & 12 & 2 & 0.12 & 0.13 \\
\hline Plasma insulin (pmol/l) & & & & & 0.41 & & & & & 0.34 & $0.21 \ddagger$ \\
\hline-15 & 129 & 11 & 132 & 14 & & 134 & 12 & 144 & 16 & & \\
\hline 0 & 118 & 9 & 120 & 13 & & 130 & 13 & 131 & 17 & & \\
\hline 15 & 402 & 37 & 418 & 53 & & 479 & 58 & 600 & 85 & & \\
\hline 30 & 729 & 73 & 645 & 76 & & 759 & 79 & 822 & 106 & & \\
\hline 60 & 1006 & 99 & 968 & 104 & & 1173 & 148 & 1064 & 127 & & \\
\hline 120 & 895 & 111 & 818 & 99 & & 1094 & 184 & 1208 & 233 & & \\
\hline IAUC insulin up to $120 \mathrm{~min}(\mathrm{pmol} / / \mathrm{per} \mathrm{min})$ & 80 & 8 & 74 & 8 & 0.40 & 95 & 12 & 95 & 13 & 0.94 & 0.51 \\
\hline
\end{tabular}

${ }^{*} P$ value obtained from paired $t$ test to compare changes from baseline (Post $v$. Pre) within the SCP $\left(P_{1}\right)$ and Control $\left(P_{2}\right)$ groups.

t $P$ value represents between-treatment comparison of changes from baseline (Post $v$. Pre), assessed by PROC MIXED ANOVA.

$\ddagger P$ value obtained from repeated measures ANOVA performed on the averages of all time points for SCP and Control to assess treatment effect. Due to significant correlations within OGTT variables, a Bonferroni correction was applied for these variables, defining level of statistical significance at $P<0.004$.

(a)

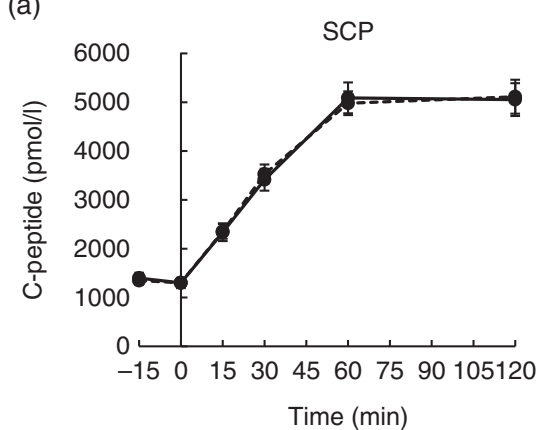

(b)

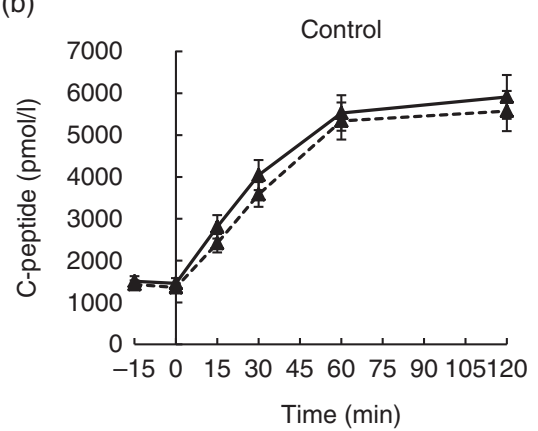

(c)

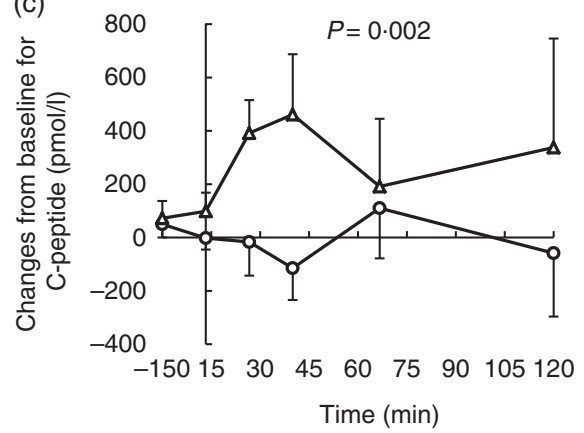

Fig. 2. Responses of plasma C-peptide at $-15,0,15,30,60$ and 120 min during the oral glucose tolerance test (OGTT) before (Pre) and after (Post) 6-week consumption of (a) strawberry and cranberry polyphenols (SCP) or (b) Control, in insulin-resistant human subjects. (c) Changes from baseline (Post $v$. Pre) in plasma C-peptide at $-15,0,15,30,60$ and 120 min during the OGTT before (Pre) and after (Post) 6-week consumption of SCP or Control in insulin-resistant human subjects. Repeated measures ANOVA showed significant difference in the changes from baseline (Post $v$. Pre) between the two groups over time during the OGTT in insulinresistant human subjects. A Bonferroni correction was applied defining level of statistical significance at $P<0.004$. Values are means $(n 41)$, with their standard errors

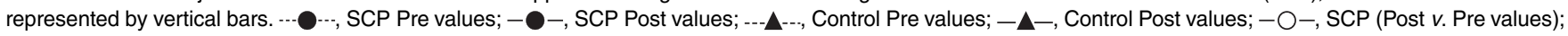
$-\triangle$ - Control (Post $v$. Pre values).

Among these, $p$-coumaric acid, $m$-coumaric acid, ferulic acid and hydroxyhippuric acid, whose absorption kinetics is presented in Fig. 5, were detected in significantly higher concentration following the consumption of SCP, relative to Control (respective AUC of 17.4 (SEM 1.8) v. 0, 85.9 (SEM 5.3) v. 0.7 (SEM $0 \cdot 3), 20 \cdot 3$ (SEM 3.9) v. 0.9 (SEM 0.4), $204 \cdot 6$ (SEM 25.4) v. 38.6 (SEM 6.9); $P<0.0001$ ). A significant negative correlation was found between plasma concentration of $p$-coumaric acid (AUC 0 $30 \mathrm{~min}$ ) and the percentage change in IAUC (before and after treatment) of the above-mentioned C-peptide response (first $30 \mathrm{~min}$ of the OGTT) $\left(P=0.0046, r^{2} 0.34\right)$. No correlations were reported between the remaining metabolites and the parameters related to insulin sensitivity and the first $30 \mathrm{~min}$ OGTT.

\section{Discussion}

This study investigated the impact of daily consumption of a $333 \mathrm{mg}$ SCP blend on insulin sensitivity in insulin-resistant, nondiabetic subjects over a period of 6 weeks. The main outcomes of this study are: (1) an improvement in insulin sensitivity, as assessed by hyperinsulinaemic-euglycaemic clamp and (2) the prevention of further compensatory insulin secretion, as shown 


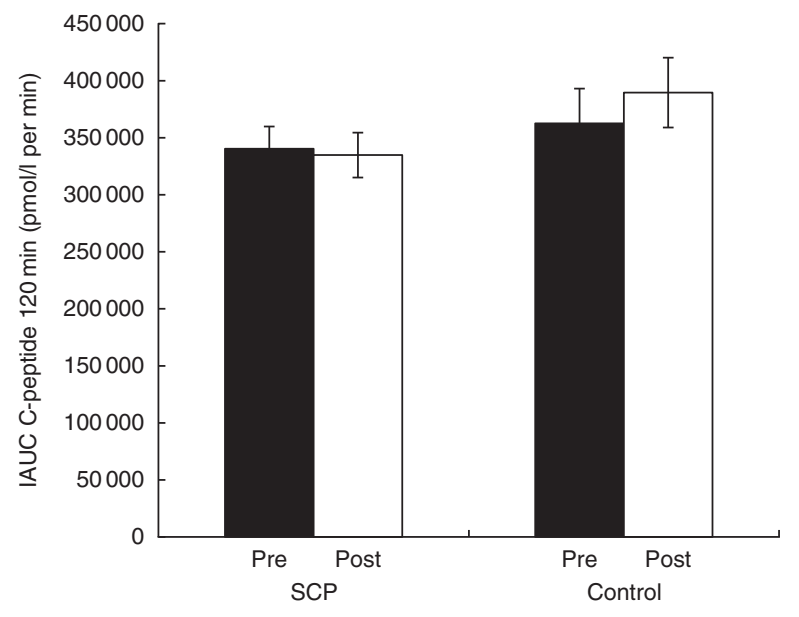

Fig. 3. Positive incremental AUC (IAUC) up to $120 \mathrm{~min}$ of the oral glucose tolerance test for C-peptide concentrations before (Pre) and after (Post) 6-week consumption of strawberry and cranberry polyphenols (SCP) or Control in insulin-resistant human subjects. Values are means $(n 41)$, with their standard errors represented by vertical bars. Paired $t$ test showed no difference in changes from baseline (Post $v$. Pre) in the SCP and Control groups. PROC MIXED for a two-way ANOVA showed no significant effect in the changes from baseline (Post $v$. Pre) between the two groups.

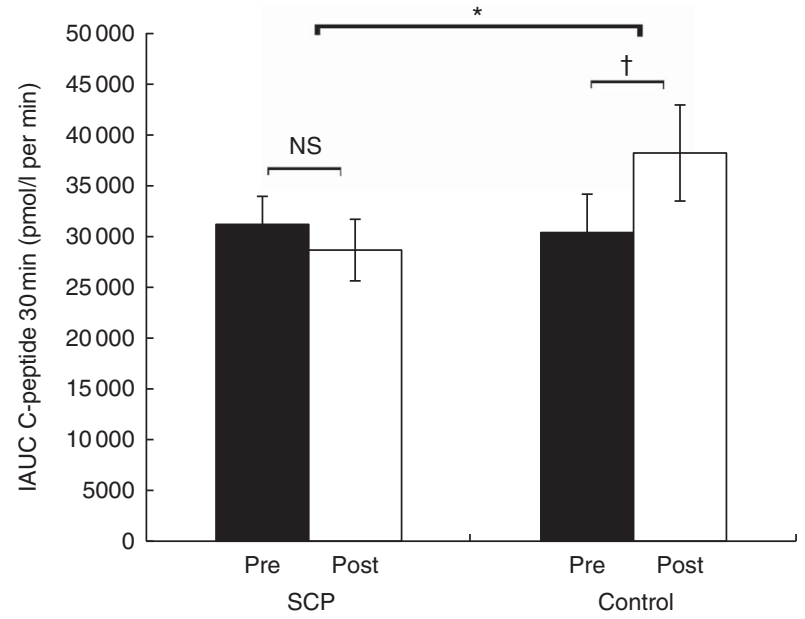

Fig. 4. Positive incremental AUC (IAUC) up to $30 \mathrm{~min}$ of the oral glucose tolerance test for C-peptide concentrations before (Pre) and after (Post) 6-week consumption of strawberry and cranberry polyphenols (SCP) or Control in insulin-resistant human subjects. Values are means $(n 41)$, with their standard errors represented by vertical bars. NS, no significant difference. Paired $t$ test for comparisons from baseline (Post $v$. Pre) within each group showed a significant increase with Control, $† P=0.003$. PROC MIXED for a two-way ANOVA showed a significant difference in the changes from baseline (Post $v$. Pre) between the two groups, ${ }^{*} P=0.002$.

by a lack of increase in the early C-peptide response during an OGTT. Analysis of plasma phenolic metabolites also revealed that the most abundant phenolic acids identified with SCP intake were $p$-coumaric acid, $m$-coumaric acid, ferulic acid and hydroxyhippuric acid. Interestingly, plasma $p$-coumaric acid was inversely related to the early $\mathrm{C}$-peptide response during OGTT.

The hyperinsulinaemic-euglycaemic clamp is the gold standard and reference technique for assessing insulin sensitivity when whole-body insulin sensitivity is the primary outcome ${ }^{(20)}$. It is a steady-state technique that requires a constant insulin infusion. It is the most reliable technique in a clinical study like ours with relatively low number of subjects. Although indirect OGTTderived indices (e.g. Matsuda index and ISI) may be of particular interest in prospective studies with large cohorts, they have potential limitations in reproducibility due to intra-individual variation in plasma glucose and insulin responses during the OGTT $^{(34)}$. With regard to the indirect indices based on fasting levels of glucose and insulin (e.g. HOMA-IR and QUICKI), they tend to assess hepatic insulin resistance rather than peripheral and whole-body insulin sensitivity ${ }^{(35)}$.

Our study demonstrates an improvement in insulin sensitivity following the consumption of SCP compared with Control. These results are in good agreement with those of Stull et al. ${ }^{(22)}$ who observed a $22 \%$ increase in insulin sensitivity, assessed with the hyperinsulinaemic-euglycaemic clamp technique, following a 6-week daily dietary supplementation with whole blueberries in obese, non-diabetic, and insulin-resistant human subjects, and those of Hokayem et al. ${ }^{(23)}$ who noted that the negative effects of fructose used to develop insulin resistance were counteracted by grape polyphenol supplementation in a double-blind controlled trial. It is noteworthy that our dose of polyphenols (a total of $333 \mathrm{mg}$ of combined SCP/d), which is a much lower dose than those used by Stull et al. ${ }^{(22)}$ (1462 mg polyphenols from blueberry powder/d) and Hokayem et al. ${ }^{(23)}$ ( $2 \mathrm{~g}$ from grape polyphenols/d), can exert a comparable effect on insulin sensitivity. The present results with SCP are also in good agreement with those reported by Edirisinghe et al. ${ }^{(36)}$ who observed a reduction in postprandial insulin response in overweight adults after a single consumption of a highcarbohydrate, moderate-fat meal with a strawberry beverage containing $10 \mathrm{~g}$ of strawberries in a freeze-dried form $(95 \mathrm{mg}$ of polyphenols). Furthermore, Park et al. ${ }^{(37)}$ observed a reduction in systolic and diastolic blood pressure concomitant with a trend to improve fasting insulin and insulin sensitivity in subjects with pre-hypertension consuming a grape seed extractcontaining beverage ( $528 \mathrm{mg}$ of polyphenols). Most of these subjects were hyperinsulinaemic or insulin resistant. This is of interest in the present study, given the evidence supporting a link between hypertension and insulin resistance through insulin mediated signalling pathways ${ }^{(38)}$ and NO production ${ }^{(39)}$. Similarly, Rodriguez-Mateos et $a l^{(40)}$ reported that endothelial function (at $1 \mathrm{~h}$ ) in healthy men increased in a dose-dependent manner up to an intake of $766 \mathrm{mg}$ polyphenols from a blueberry drink and reached a plateau at higher doses. Therefore, the present results together with those from the three latter studies suggest that polyphenol doses lower than $800 \mathrm{mg}$ may offer metabolic benefits. Of note, the beneficial effect of SCP on insulin sensitivity observed in the present study cannot be explained by variations in energy and macronutrient intake, body weight or body fat mass since no changes in these parameters were seen between the two groups.

The progression from normal glucose tolerance to type 2 diabetes is characterised by both an increase in insulin resistance and a decrease in insulin secretion caused by $\beta$-cell dysfunction. Insulin resistance is defined as decreased tissue sensitivity to insulin to stimulate glucose uptake and utilisation. 
Table 4. Lipid profile, inflammatory and thrombogenic markers, and oxidative status before and after 6-week consumption of strawberry and cranberry polyphenols (SCP) or Control in insulin-resistant human subjects

(Mean values with their standard errors)

\begin{tabular}{|c|c|c|c|c|c|c|c|c|c|c|c|}
\hline \multirow[b]{3}{*}{ Variables } & \multicolumn{4}{|c|}{$\operatorname{SCP}(n 20)$} & \multirow[b]{3}{*}{$P_{1}^{*}$} & \multicolumn{4}{|c|}{ Control ( $n 21)$} & \multirow[b]{3}{*}{$P_{2}{ }^{*}$} & \multirow[b]{3}{*}{$P+$} \\
\hline & \multicolumn{2}{|c|}{ Pre } & \multicolumn{2}{|c|}{ Post } & & \multicolumn{2}{|c|}{ Pre } & \multicolumn{2}{|c|}{ Post } & & \\
\hline & Mean & SEM & Mean & SEM & & Mean & SEM & Mean & SEM & & \\
\hline \multicolumn{12}{|l|}{ Cholesterol (mmol/l) } \\
\hline Total & 5.70 & $0 \cdot 17$ & 5.60 & 0.19 & 0.52 & 5.37 & 0.22 & 5.45 & 0.20 & 0.45 & 0.33 \\
\hline $\mathrm{HDL}$ & 1.25 & 0.05 & 1.26 & 0.06 & 0.68 & 1.33 & 0.05 & 1.37 & 0.06 & 0.10 & 0.40 \\
\hline LDL & 3.52 & 0.17 & $3 \cdot 51$ & 0.17 & 0.97 & $3 \cdot 20$ & 0.15 & $3 \cdot 37$ & 0.17 & 0.05 & 0.32 \\
\hline Total cholesterol:HDL-cholesterol ratio & 4.76 & 0.27 & 4.62 & 0.24 & $0 \cdot 16$ & $4 \cdot 12$ & 0.20 & 4.08 & 0.17 & 0.60 & 0.41 \\
\hline TAG (mmol/l) & 2.03 & 0.24 & $1 \cdot 82$ & 0.21 & 0.06 & 1.73 & 0.26 & 1.56 & 0.18 & 0.07 & 0.99 \\
\hline hsCRP (mg/l)‡ & 3.6 & 0.7 & $3 \cdot 0$ & 0.6 & 0.13 & $5 \cdot 4$ & $2 \cdot 9$ & 3.0 & 0.6 & 0.69 & 0.53 \\
\hline TNF- $\alpha(\mathrm{ng} / \mathrm{l}) \S$ & 4.4 & 0.4 & 4.0 & 0.4 & 0.06 & $4 \cdot 3$ & 0.2 & 4.0 & 0.3 & 0.32 & 0.69 \\
\hline IL-6 (ng/l)§ & 4.9 & 0.4 & $4 \cdot 8$ & 0.6 & 0.99 & $5 \cdot 6$ & 1.0 & 4.9 & 0.8 & 0.24 & 0.23 \\
\hline HMW adiponectin $(\mu \mathrm{g} / \mathrm{ml}) \S$ & 6.83 & $1 \cdot 10$ & 5.91 & 0.93 & 0.15 & $7 \cdot 15$ & 1.15 & 6.73 & 1.20 & 0.61 & 0.65 \\
\hline PAl- $1 \times 10^{3}(\mathrm{ng} / \mathrm{l}) \S$ & 30.5 & $2 \cdot 7$ & 28.5 & $3 \cdot 1$ & 0.46 & $30 \cdot 0$ & 2.5 & $27 \cdot 3$ & 3.5 & 0.41 & 0.87 \\
\hline RANTES $(\mu \mathrm{g} / \mathrm{l}) \ddagger$ & $3 \cdot 21$ & 0.39 & 3.15 & 0.44 & 0.86 & 3.06 & 0.40 & $2 \cdot 77$ & 0.37 & 0.53 & 0.71 \\
\hline Oxidised-LDL (U/l)‡ & 96.5 & $6 \cdot 2$ & $92 \cdot 9$ & $5 \cdot 6$ & 0.14 & $79 \cdot 7$ & 5.4 & $80 \cdot 2$ & 4.8 & 0.83 & 0.22 \\
\hline FRAP $\left(\mu \mathrm{M} \mathrm{Fe} e^{2+} / l\right) \ddagger$ & 1191 & 58 & 1237 & 65 & 0.37 & 1135 & 36 & 1189 & 41 & 0.07 & 0.70 \\
\hline
\end{tabular}

hsCRP, high-sensitivity C-reactive protein; HMW, high molecular weight; PAl-1, plasminogen activator inhibitor-1; RANTES, regulated on activation, normal T cell expressed and secreted; FRAP, ferric reducing antioxidant power.

* $P$ value obtained from paired $t$ test to compare changes from baseline (Post $v$. Pre) within the SCP $\left(P_{1}\right)$ and Control $\left(P_{2}\right)$ groups.

$\dagger P$ value represents between-treatment comparison of changes from baseline (Post $v$. Pre), assessed by PROC MIXED ANOVA. Due to significant correlations between lipid and

oral glucose tolerance test variables, a Bonferroni correction was applied for these variables, defining level of statistical significance at $P<0.004$.

$\ddagger n$ 38-39 (SCP $n$ 18-20; Control $n$ 18-21).

$\S n 33$ (SCP $n$ 15; Control $n$ 18).

(a)

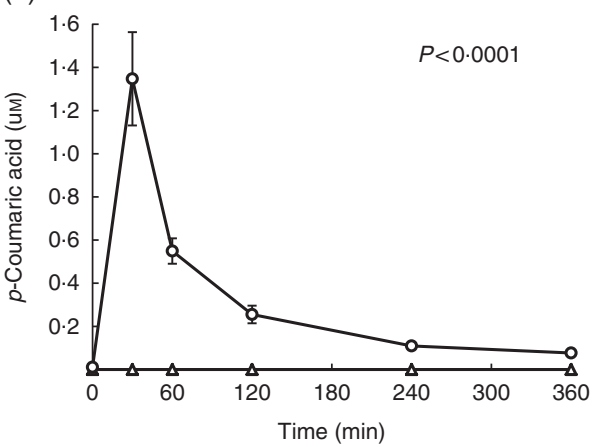

(c)

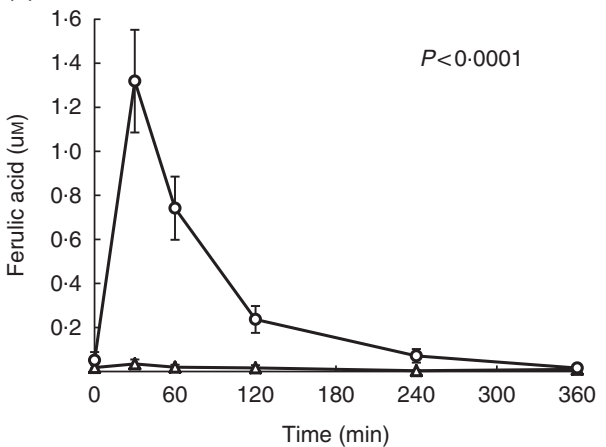

(b)

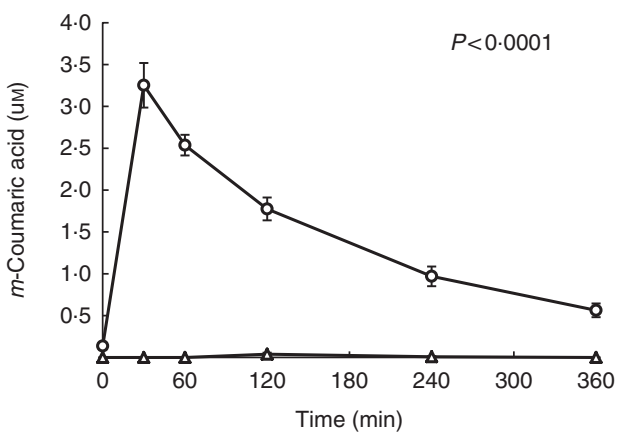

(d)

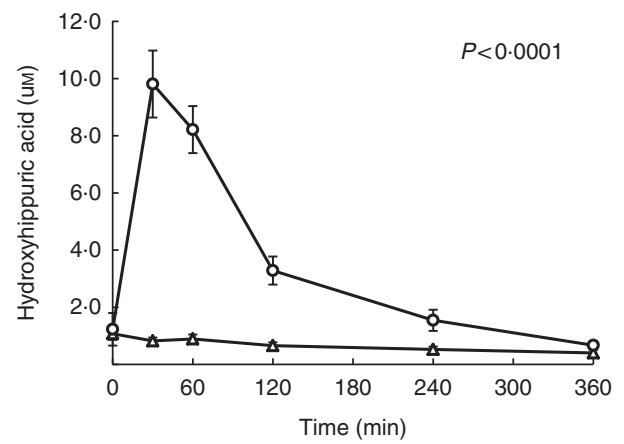

Fig. 5. Evolution of post-ingestion plasma concentrations of phenolic metabolites. (a) p-coumaric acid, (b) $m$-coumaric acid, (c) ferulic acid, (d) hydroxyhippuric acid. Values are mean of replicates $(n 17)$, with their standard errors. Welch's $t$ test (correcting for unequal variance) when data were assumed to be normally distributed and the non-parametric Mann-Whitney test otherwise, showed significantly higher concentrations of phenolic metabolites following the consumption of strawberry and cranberry polyphenols (SCP) relative to Control. A Bonferroni correction $(P<0.0025)$ was performed. $P<0.0001$ for each phenolic metabolite. $-O-$, SCP; $-\triangle-$, Control. 
In the early stages of insulin resistance, plasma glucose is maintained at normal levels by a compensatory increase in insulin secretion, the first abnormality being an increase in firstphase insulin secretion by pancreatic $\beta$-cells ${ }^{(41)}$. But when $\beta$-cell compensation fails, fasting plasma glucose levels rise (IFG), leading to IGT and eventually type 2 diabetes $^{(42)}$. In the context of the present study, the beverage rich in polyphenols prevented a further elevation in early-phase insulin release, as indicated by C-peptide levels, and prevented the overall increase of insulin secretion, suggesting that the improvement in insulin sensitivity after consumption of the SCP beverage may have precluded a further compensatory increase in insulin secretion. A reducing effect of a polyphenol-rich cranberry extract has already been observed on C-peptide levels in highfat/high-sucrose-fed mice ${ }^{(43)}$. However, in humans, to the best of our knowledge, this is the first report showing a beneficial effect of a SCP extract on C-peptide response during an OGTT.

In this study, we found differences between the SCP and the Control groups in the plasma levels of four polyphenolic compounds and metabolites ( $p$-coumaric acid, $m$-coumaric acid, ferulic acid, hydroxyhippuric acid). These levels were found in the same low micromolar range as that reported by Feliciano et $a l^{(44)}$ and Park et al. ${ }^{(37)}$, where physiological and metabolic bioactivity is probable ${ }^{(45)}$. Particularly of interest, an increased plasma concentration of $p$-coumaric acid during the $30 \mathrm{~min}$ post-consumption was found to significantly correlate with a reduced secretion of C-peptide compared with the Control during the early phase of the OGTT response $\left(P=0.0046, r^{2} 0.34\right)$. Although a direct effect of $p$-coumaric acid on insulin sensitivity cannot be confirmed by these results, this compound was recently found to stimulate AMP-activated protein kinase phosphorylation, leading to increased glucose uptake in L6 myocytes ${ }^{(46)}$. $p$-Coumaric acid was also found to improve glucose uptake in vitro through synergistic interactions with a commercial oral hypoglycaemic drug (thiazolidinedione $)^{(47)}$. Moreover, since only $5-10 \%$ of ingested phenolic compounds are assumed to be absorbed ${ }^{(48)}$, a large amount proceeds to the large intestine (especially polymeric forms such as proanthocyanidins) where they can exert an activity. Indeed, we recently showed that cranberry polyphenols can improve insulin sensitivity in high-fat-fed mice, leading to reduced inflammation in both intestinal and hepatic tissues, through modulation of gut microbiota ${ }^{(43)}$. In this latter study, changes in gut microbiota were detected following 5 weeks of supplementation, suggesting that 6 weeks of supplementation in humans was very likely a period long enough to modulate their intestinal microbiota. SCP may also improve insulin sensitivity by increasing insulin signalling and glucose transport in skeletal muscle cells. Indeed, Nizamutdinova et al. ${ }^{(17)}$ showed that anthocyanins administrated by gavage can improve insulin signalling by stimulating tyrosine phosphorylation of the insulin receptors and by increasing the expression of GLUT4 in muscle of streptozotocin-diabetic rats.

Whereas our study provides evidence for the beneficial effect of SCP treatment on insulin sensitivity, this finding was not associated with a decrease in other markers of cardiovascular risk. Nutritional studies are somewhat contradictory regarding the effects of strawberries and cranberries on cardiometabolic markers such as plasma lipids, oxidative stress, antioxidant capacity and inflammation. Indeed, lipid changes seen in our study contrast with those reported by Basu et al. ${ }^{(7)}$ and Lee et al. ${ }^{(49)}$ who observed a decrease in total cholesterol and LDLcholesterol in human subjects consuming either freeze-dried strawberry powder ${ }^{(8)}$ or cranberry extract ${ }^{(49)}$. Similarly, our findings on inflammatory and oxidative markers do not agree with those of Moazen et al. ${ }^{(50)}$ who observed a decrease in plasma CRP and oxidised-LDL after administration of freezedried strawberries, and those of Ruel et al..$^{(9,11,51)}$ who observed an increase of plasma HDL-cholesterol, antioxidant capacity and a decrease in oxidised-LDL after consumption of lowenergy cranberry drink. Additionally, some clinical studies investigating the effects of approximately $300 \mathrm{mg}$ polyphenols from freeze-dried strawberry powder reported reducing effects on fasting oxidised LDL for 6 weeks ${ }^{(9)}$, and postprandial CRP and IL-6 $6^{(36)}$ in an overweight, hyperlipidaemic population. However, other clinical studies using freeze-dried strawberry powder or reduced-energy cranberry juice have demonstrated, as in our study, a lack of effect on antioxidant status ${ }^{(52)}$, on CRP, IL-6 and TNF- $\alpha^{(52,53)}$.

Discrepancies between our results on lipids and inflammatory and oxidative markers and those of the above-cited studies $^{(8,12,36,49-51)}$ may stem from the delivery form of strawberry (freeze-dried form $v$. SCP extract) or cranberry (juice, dried fruit forms or SCP extract), the experimental design (acute $v$. longer term), the polyphenol dose or the population studied. In the present study, the SCP were a SCP enriched extract providing $333 \mathrm{mg} / \mathrm{d}$ of polyphenols and was devoid of fibres, sugars, minerals and vitamins. On the one hand, this amount of polyphenols, which corresponds to the quantity supplied by about $120 \mathrm{~g}$ of fresh fruits, is equivalent to approximately onefourth the level of polyphenols present in the freeze-dried strawberry powder used by Basu et al. ${ }^{(7)}$ and Moazen et al. ${ }^{(50)}$ (1001-2006 mg polyphenols/d) and in the low-energy cranberry juice used in previous studies ${ }^{(9-12,15,51)}$. On the other hand, the key difference between the SCP extract used in the present study and these other sources of polyphenols is the lack of fermentable fibres. It is well recognised that strawberries ${ }^{(54)}$ and cranberries ${ }^{(55)}$ contain significant amounts of fermentable soluble and insoluble fibres that may reduce lipids, inflammatory and oxidative markers ${ }^{(56)}$. Therefore, the lack of dietary fibre in our extract is among the plausible explanations for the absence of an SCP effect on lipids, inflammatory and oxidative markers in the current study. Moreover, the present results and those from a study by Hokayem et al. ${ }^{(23)}$ who used a berry (grape) polyphenol extract, suggest that the various phenol compounds present in berries may specifically improve insulin sensitivity in humans.

The participants in this study were insulin resistant and included both sexes and a relatively broad age range (40-70 years). Given the free-living nature of the study, the results can most likely apply to an overweight adult pre-diabetic population. Despite the parallel arm design and polyphenol content of the beverage used, the present study did allow sufficient power to detect statistical differences on the primary endpoint of insulin sensitivity. However, this study was not sufficiently powered to detect differences on secondary 
endpoints, in particular hsCRP, TNF- $\alpha$ and oxidised-LDL. Yet, it would have been interesting to obtain muscle and adipose tissue biopsies to test whether SCP reduced inflammation in these tissues. Such biopsies may have also allowed us to ascertain the identity of the molecules involved in cellular insulin signalling. Nonetheless, considering the robust nature of our randomised, controlled, double-blind study, it is likely that the consumption of SCP resulted in the improvement in the hyperinsulinaemiceuglycaemic clamp insulin sensitivity and OGTT parameters. Finally, since this is a proof-of-concept study, it will be necessary to consider longer-term interventions in larger populations of subjects to confirm the results and expand upon the potential role of SCP in preventing or delaying the onset of type 2 diabetes.

In conclusion, our data indicate that 6-week consumption of $333 \mathrm{mg}$ polyphenols from strawberries and cranberries may improve insulin sensitivity and prevent an increase in compensatory insulin secretion without affecting plasma lipids, CRP, pro-inflammatory cytokines and antioxidant capacity. Controlled dose-response trials are needed to ascertain the lower and upper range of activity of these polyphenols, as well as larger and longer-term studies.

\section{Acknowledgements}

The authors are grateful to Marie-Christine Dubé, Louise Rhéaume, Valérie-Ève Julien, Marie Tremblay and Camille Lambert, from the Diabetes Research Unit (Laval University Health Center of Quebec) who helped in performing hyperinsulinaemic-euglycaemic clamps and Steeve Larouche and Danielle Aubin, the nurses who performed OGTT (INAF, Laval University). The authors also thank Hélène Crépeau from Laval University, for help with statistical analysis and the subjects who participated in this study. Finally, the authors acknowledge Jean-Paul-Houle Funds, Diabète Québec and INAF for scholarships. The present study was supported by the Consortium de recherche et innovations en bioprocédés industriels au Québec, Atrium Innovations Inc. and Nutra Canada. Parts of this study were presented in abstract form at the 17th Annual Canadian Diabetes Association/Canadian Society of Endocrinology and Metabolism (CDA/CSEM) Professional Conference \& Annual Meetings, Winnipeg, Manitoba, Canada, 22-25 October 2014.

The authors' contributions were as follows: S. J. W., Y. D., A. M. and H. J. designed research. J. M. and H. J. planned the study. M. P. and J. M. conducted research. M. P. and A. S. M.-L. performed statistical analysis. M. P., S. J. W., Y. D., A. M. and H. J wrote the paper. S. D. performed polyphenol determinations. H. J. had primary responsibility for the final content. M. P., A. S. M.-L., S. J. W., Y. D., J. M., G. P., S. D., A. M. and H. J. contributed to the discussion and data interpretation. All authors have read and approved the final manuscript. The study funders had no role in the study design or in the collection, analysis, interpretation of data and decision to publish. The authors have sole responsibility for the manuscript content.

None of the authors has any conflicts of interest to declare.

\section{Supplementary material}

For supplementary material/s referred to in this article, please visit https://doi.org/10.1017/S0007114517000393

\section{References}

1. International Diabetes Federation (2013) IDF Diabetes Atlas, 6th ed. pp. 11-12. Brussels: International Diabetes Federation.

2. Anderson JW, Kendall CW \& Jenkins DJ (2003) Importance of weight management in type 2 diabetes: review with meta analysis of clinical studies. J Am Coll Nutr 22, 331-339.

3. Jeon CY, Lokken RP, Hu FB, et al. (2007) Physical activity of moderate intensity and risk of type 2 diabetes: a systematic review. Diabetes Care 30, 744-752.

4. Wellen KE \& Hotamisligil GS (2005) Inflammation, stress, and diabetes. J Clin Invest 115, 1111-1119.

5. Mursu J, Virtanen JK, Tuomainen TP, et al. (2014) Intake of fruit, berries, and vegetables and risk of type 2 diabetes in Finnish men: the Kuopio Ischaemic Heart Disease Risk Factor Study. Am J Clin Nutr 99, 328-333.

6. Basu A, Fu DX, Wilkinson M, et al. (2010) Strawberries decrease atherosclerotic markers in subjects with metabolic syndrome. Nutr Res 30, 462-469.

7. Basu A, Wilkinson M, Penugonda K, et al. (2009) Freeze-dried strawberry powder improves lipid profile and lipid peroxidation in women with metabolic syndrome: baseline and post intervention effects. Nutr J 8, 43.

8. Burton-Freeman B, Linares A, Hyson D, et al. (2010) Strawberry modulates LDL oxidation and postprandial lipemia in response to high-fat meal in overweight hyperlipidemic men and women. J Am Coll Nutr 29, 46-54.

9. Ruel G, Pomerleau S, Couture P, et al. (2006) Favourable impact of low-calorie cranberry juice consumption on plasma HDL-cholesterol concentrations in men. $\mathrm{Br} J$ Nutr 96, 357-364.

10. Basu A, Betts NM, Ortiz J, et al. (2011) Low-energy cranberry juice decreases lipid oxidation and increases plasma antioxidant capacity in women with metabolic syndrome. Nutr Res 31, 190-196.

11. Ruel G, Pomerleau S, Couture P, et al. (2005) Changes in plasma antioxidant capacity and oxidized low-density lipoprotein levels in men after short-term cranberry juice consumption. Metabolism 54, 856-861.

12. Afrin S, Gasparrini M, Forbes-Hernandez TY, et al. (2016) Promising health benefits of the strawberry: a focus on clinical studies. J Agric Food Chem 64, 4435-4449.

13. Giampieri F, Forbes-Hernandez TY, Gasparrini M, et al. (2015) Strawberry as a health promoter: an evidence based review. Food Funct 6, 1386-1398.

14. Novotny JA, Baer DJ, Khoo C, et al. (2015) Cranberry juice consumption lowers markers of cardiometabolic risk, including blood pressure and circulating C-reactive protein, triglyceride, and glucose concentrations in adults. J Nutr $\mathbf{1 4 5}$, 1185-1193.

15. Szajdek A \& Borowska EJ (2008) Bioactive compounds and health-promoting properties of berry fruits: a review. Plant Foods Hum Nutr 63, 147-156.

16. Hanhineva K, Törrönen R, Bondia-Pons I, et al. (2010) Impact of dietary polyphenols on carbohydrate metabolism. Int J Mol Sci 11, 1365-1402.

17. Nizamutdinova IT, Jin YC, Chung JI, et al. (2009) The antidiabetic effect of anthocyanins in streptozotocin-induced diabetic rats through glucose transporter 4 regulation and prevention of insulin resistance and pancreatic apoptosis. $\mathrm{Mol}$ Nutr Food Res 53, 1419-1429.

18. Denis MC, Desjardins Y, Furtos A, et al. (2015) Prevention of oxidative stress, inflammation and mitochondrial dysfunction in the intestine by different cranberry phenolic fractions. Clin Sci (Lond) 128, 197-212. 
19. Takikawa M, Inoue S, Horio F, et al. (2010) Dietary anthocyanin-rich bilberry extract ameliorates hyperglycemia and insulin sensitivity via activation of AMP-activated protein kinase in diabetic mice. $J$ Nutr 140, 527-533.

20. DeFronzo RA, Tobin JD \& Andres R (1979) Glucose clamp technique: a method for quantifying insulin secretion and resistance. Am J Physiol 237, E214-E223.

21. Antuna-Puente B, Disse E, Rabasa-Lhoret R, et al. (2011) How can we measure insulin sensitivity/resistance? Diabetes Metab 37, 179-188.

22. Stull AJ, Cash KC, Johnson WD, et al. (2010) Bioactives in blueberries improve insulin sensitivity in obese, insulinresistant men and women. J Nutr 140, 1764-1768.

23. Hokayem M, Blond E, Vidal H, et al. (2013) Grape polyphenols prevent fructose-induced oxidative stress and insulin resistance in first-degree relatives of type 2 diabetic patients. Diabetes Care 36, 1454-1461.

24. Dudonné S, Dubé P, Pilon G, et al. (2014) Modulation of strawberry/cranberry phenolic compounds glucuronidation by co-supplementation with onion: characterization of phenolic metabolites in rat plasma using an optimized $\mu \mathrm{SPE}-$ UHPLC-MS/MS method. J Agric Food Chem 62, 3244-3256.

25. Expert Committee on the Diagnosis and Classification of Diabetes Mellitus (2003) Follow-up report on the diagnosis of diabetes mellitus. Diabetes Care 26, 3160-3167.

26. Labonte ME, Cyr A, Baril-Gravel L, et al. (2012) Validity and reproducibility of a web-based, self-administered food frequency questionnaire. Eur J Clin Nutr 66, 166-173.

27. Piche ME, Weisnagel SJ, Corneau L, et al. (2005) Contribution of abdominal visceral obesity and insulin resistance to the cardiovascular risk profile of postmenopausal women. Diabetes 54, 770-777.

28. Richterich R \& Dauwalder H (1971) Determination of plasma glucose by hexokinase- glucose-6-phosphate dehydrogenase method. Schweiz Med Wochenschr 101, 615-618.

29. Desbuquois B \& Aurbach GD (1971) Use of polyethylene glycol to separate free and antibody-bound peptide hormones in radioimmunoassays. J Clin Endocrinol Metab 33, 732-738.

30. Couillard C, Després JP, Lamarche B, et al. (2001) Effects of endurance exercise training on plasma HDL cholesterol levels depend on levels of triglycerides: evidence from men of the Health, Risk Factors, Exercise Training and Genetics (HERITAGE) Family Study. Arterioscler Thromb Vasc Biol 21, 1226-1232.

31. Piché ME, Lemieux S, Weisnagel SJ, et al. (2005) Relation of high-sensitivity C-reactive protein, interleukin-6, tumor necrosis factor-alpha, and fibrinogen to abdominal adipose tissue, blood pressure, and cholesterol and triglyceride levels in healthy postmenopausal women. Am J Cardiol 96, 92-97.

32. Rubió L, Serra A, Chen CY, et al. (2014) Effect of the co-occurring components from olive oil and thyme extracts on the antioxidant status and its bioavailability in an acute ingestion in rats. Food Funct 5, 740-747.

33. Ouellet V, Marois J, Weisnagel SJ, et al. (2007) Dietary cod protein improves insulin sensitivity in insulin-resistant men and women: a randomized controlled trial. Diabetes Care 30, 2816-2821.

34. Balion CM, Raina PS, Gerstein HC, et al. (2007) Reproducibility of impaired glucose tolerance (IGT) and impaired fasting glucose (IFG) classification: a systematic review. Clin Chem Lab Med 45, 1180-1185.

35. Borai A, Livingstone C, Kaddam I, et al. (2011) Selection of the appropriate method for the assessment of insulin resistance. BMC Med Res Methodol 11, 158-167.
36. Edirisinghe I, Banaszewski K, Cappozzo J, et al. (2011) Strawberry anthocyanin and its association with postprandial inflammation and insulin. Br J Nutr 106, 913-922.

37. Park E, Edirisinghe I, Choy YY, et al. (2016) Effects of grape seed extract beverage on blood pressure and metabolic indices in individuals with pre-hypertension: a randomised, double-blinded, two-arm parallel, placebo-controlled trial. $\mathrm{BrJ}$ Nutr 115, 226-238.

38. Addison S, Stas S, Hayden MR, et al. (2008) Insulin resistance and blood pressure. Curr Hypertens Rep 10, 319-325.

39. Kim JA, Montagnani M, Koh KK, et al. (2006) Reciprocal relationships between insulin resistance and endothelial dysfunction: molecular and pathophysiological mechanisms. Circulation 113, 1888-1904.

40. Rodriguez-Mateos A, Rendeiro C, Bergillos-Meca T, et al. (2013) Intake and time dependence of blueberry flavonoidinduced improvements in vascular function: a randomized, controlled, double-blind, crossover intervention study with mechanistic insights into biological activity. Am J Clin Nutr 98, 1179-1191.

41. Kahn SE, Prigeon RL, McCulloch DK, et al. (1993) Quantification of the relationship between insulin sensitivity and betacell function in human subjects. Evidence for a hyperbolic function. Diabetes 42, 1663-1672.

42. Pratley RE \& Weyer C (2002) Progression from IGT to type 2 diabetes mellitus: the central role of impaired early insulin secretion. Curr Diab Rep 2, 242-248.

43. Anhê FF, Roy D, Pilon G, et al. (2015) A polyphenol-rich cranberry extract protects from diet-induced obesity, insulin resistance and intestinal inflammation in association with increased Akkermansia spp. population in the gut microbiota of mice. Gut 64, 872-883.

44. Feliciano RP, Boeres A, Massacessi L, et al. (2016) Identification and quantification of novel cranberry-derived plasma and urinary (poly)phenols. Arch Biochem Biophys 599, 31-41.

45. Scheepens A, Tan K \& Paxton JW (2010) Improving the oral bioavailability of beneficial polyphenols through designed synergies. Genes Nutr 5, 75-87.

46. Yoon SA, Kang SI, Shin HS, et al. (2013) p-Coumaric acid modulates glucose and lipid metabolism via AMP-activated protein kinase in L6 skeletal muscle cells. Biochem Biophys Res Commun 432, 553-557.

47. Prabhakar PK \& Doble M (2011) Interaction of cinnamic acid derivatives with commercial hypoglycemic drugs on 2-deoxyglucose uptake in 3T3-L1 adipocytes. J Agric Food Chem 59, 9835-9844.

48. Cardona F, Andrés-Lacueva C, Tulipani S, et al. (2013) Benefits of polyphenols on gut microbiota and implications in human health. J Nutr Biochem 24, 1415-1422.

49. Lee IT, Chan YC, Lin CW, et al. (2008) Effect of cranberry extracts on lipid profiles in subjects with type 2 diabetes. Diabet Med 25, 1473-1477.

50. Moazen S, Amani R, Homayouni Rad A, et al. (2013) Effects of freeze-dried strawberry supplementation on metabolic biomarkers of atherosclerosis in subjects with type 2 diabetes: a randomized double-blind controlled trial. Ann Nutr Metab 63, 256-264

51. Ruel G, Pomerleau S \& Couture P (2008) Low-calorie cranberry juice supplementation reduces plasma oxidized LDL and cell adhesion molecule concentrations in men. Br J Nutr 99, 352-359.

52. Zunino SJ, Parelman MA, Freytag TL, et al. (2012) Effects of dietary strawberry powder on blood lipids and inflammatory markers in obese human subjects. Br J Nutr 108, 900-909.

53. Ellis CL, Edirisinghe I, Kappagoda T, et al. (2011) Attenuation of meal-induced inflammatory and thrombotic responses in 
overweight men and women after 6-week daily strawberry (Fragaria) intake - a randomized placebo-controlled trial. $J$ Atheroscler Thromb 18, 318-327.

54. Quirós-Sauceda AE, Palafox-Carlos H, Sáyago-Ayerdi SG, et al. (2014) Dietary fiber and phenolic compounds as functional ingredients: interaction and possible effect after ingestion. Food Funct 5, 1063-1010.
55. Hotchkiss AT Jr, Nuñez A, Strahan GD, et al. (2015) Cranberry xyloglucan structure and inhibition of Escherichia coli adhesion to epithelial cells. J Agric Food Chem 63, 5622-5633.

56. Erkkilä AT \& Lichtenstein AH (2006) Fiber and cardiovascular disease risk: how strong is the evidence? J Cardiovasc Nurs 21, 3-8. 